\title{
Microstructure evolution in grey cast iron during directional solidification
}

\author{
Xian-fei Ding ${ }^{1,2)}$, Xiao-zheng Li $^{3)}$, Qiang Feng ${ }^{2,3)}$, Warkentin Matthias ${ }^{4)}$, and Shi-yao Huang ${ }^{5)}$ \\ 1) National Center for Materials Service Safety, University of Science and Technology Beijing, Beijing 100083, China \\ 2) Beijing Key Laboratory of Special Melting and Preparation of High-end Metal, University of Science and Technology Beijing, Beijing 100083, China \\ 3) State Key Laboratory for Advanced Metals and Materials, University of Science and Technology Beijing, Beijing 100083, China \\ 4) Ford Research \& Advanced Engineering Europe, AA-FFA-1, Süsterfeldstr. 200 Aachen, 52072, Germany \\ 5) Materials and Process Research, Ford Motor Research and Engineering Center, Nanjing 211100 , China \\ (Received: 2 March 2017; revised: 22 March 2017; accepted: 24 March 2017)
}

\begin{abstract}
The solidification characteristics and microstructure evolution in grey cast iron were investigated through Jmat-Pro simulations and quenching performed during directional solidification. The phase transition sequence of grey cast iron was determined as $\mathrm{L} \rightarrow \mathrm{L}+\gamma \rightarrow \mathrm{L}+$ $\gamma+\mathrm{G} \rightarrow \gamma+\mathrm{G} \rightarrow \mathrm{P}\left(\alpha+\mathrm{Fe}_{3} \mathrm{C}\right)+\alpha+\mathrm{G}$. The graphite can be formed in three ways: directly nucleated from liquid through the eutectic reaction $(\mathrm{L} \rightarrow \gamma+\mathrm{G})$, independently precipitated from the oversaturated $\gamma$ phase $(\gamma \rightarrow \gamma+\mathrm{G})$, and produced via the eutectoid transformation $(\gamma \rightarrow \mathrm{G}+\alpha)$. The area fraction and length of graphite as well as the primary dendrite spacing decrease with increasing cooling rate. Type-A graphite is formed at a low cooling rate, whereas a high cooling rate results in the precipitation of type-D graphite. After analyzing the graphite precipitation in the as-cast and transition regions separately solidified with and without inoculation, we concluded that, induced by the inoculant addition, the location of graphite precipitation changes from mainly the $\gamma$ interdendritic region to the entire $\gamma$ matrix. It suggests that inoculation mainly acts on graphite precipitation in the $\gamma$ matrix, not in the liquid or at the solid-liquid front.
\end{abstract}

Keywords: directional solidification; grey cast iron; phase transition; graphite

\section{Introduction}

Because of its good thermal properties with balanced strength, grey cast iron is one of the most widely used materials in automobile components such as brake rotors and flywheels. A conventional casting process is usually used to produce the grey iron components directly. Because the mechanical properties as well as the thermal properties are closely related to the as-cast microstructures, microstructure control during cast processing is a major challenge for improving the properties of grey cast iron.

Solidification behavior can provide useful information for the casting process of grey cast irons. The as-cast microstructures of the cast iron, which are governed by the solidification process and solid-state transformation, are dependent on the solidification processing parameters. The solidification microstructure characteristic of grey cast iron has been extensively investigated under different solidification conditions. Hejazi et al. reported that the morphology of graphite in grey cast iron is related to the cooling rate [1]. Behnam et al. established the relationship between cooling rate and the primary/secondary dendrite arm spacing [2]. Oloyede et al. investigated the microstructure and properties of grey cast iron under rapid solidification [3]. However, the scopes of most of these investigations on solidification microstructure characteristics have not included the solidification procedure or phase transformations during cooling, resulting in an incomplete understanding of the solidification behavior. Although a few early works focused on the complicated solidification mechanisms of cast iron based on the interruption by rapid quenching at various stages of natural solidification [4-5], this technique was inefficient and complicated because a number of the heated cast irons were independently quenched at various temperatures, leading to an unclear understanding of the formation procedure of the solidification microstructure. A deep understanding of the solidification behavior and phase transition is still required to enable control of the as-cast microstructure of grey cast iron.

\footnotetext{
Corresponding author: Xian-fei Ding E-mail: xfding@ustb.edu.cn

(C) The Author(s) 2017. This article is published with open access at link.springer.com
}

Springer 
As one of the effective methods to investigate the solidification behavior and characteristics of cast irons, Bridgman directional solidification preserves the solidification microstructures formed at continuous temperatures through single quenching performed during directional solidification [6-7]. The cooling rate can be precisely controlled through modification of the withdrawal rate, and the microstructures around the solid-liquid interface are frozen by quenching because of the existence of an axial temperature gradient. After a systematic investigation of the quenched microstructures, the solidification and phase transition process can be investigated by analyzing the microstructure evolution in quenched mushy zones along the directional solidification direction.

The aim of this work is to investigate the solidification behavior and characteristics in grey cast iron through Jmat-Pro calculations as well as microstructure observations after Bridgman directional solidification. The phase transition during cooling and the microstructure evolution of both graphite and the matrix were investigated.

\section{Experimental}

The as-cast grey cast iron with the chemical composition listed in Table 1 was taken from a flywheel component produced by sand-casting with inoculant. The sample was cut into bars with a diameter of $6.8 \mathrm{~mm}$ and a height of $85 \mathrm{~mm}$. The bars were re-melted and directionally solidified using the Bridgman method at the withdrawal rates $(V)$ of 5 , 10 , and $20 \mu \mathrm{m} / \mathrm{s}$. The temperature gradient $(G)$ was approximately $10 \mathrm{~K} / \mathrm{mm}$ during directional solidification. To investigate the phase transitions of the grey cast iron, quenching was carried out immediately after directional solidification to a constant length of $45 \mathrm{~mm}$. The directionally solidified bars were sectioned longitudinally and then polished using standard metallographic techniques. The graphite microstructures were analyzed by optical microscopy (OM, ZEISS Axio Imager A2m) and field-emission scanning electron microscopy (FESEM) using a Zeiss SUPRA 55 in backscattered electron (BSE) imaging mode; the matrix microstructures were observed by FESEM after the specimens were etched with the $4 \mathrm{vol} \%$ nital solution. Statistical analysis on the area fraction of graphite in the directional solidification region was conducted using the Image J software, and more than ten images at a magnification of $200 \times$ were used to calculate the average value and error for final results. After measurements on more than three images, the length of graphite was determined by the average length value of three longest curved graphite flakes. The primary dendrite spacing was measured on the basis of the distances between the two closest primary arm axes in the quenched mushy zone by examination of the OM microstructures in longitudinal sections of directionally solidified bars. The Jmat-Pro software was used to calculate the phase transition of the grey cast iron under equilibrium conditions at high temperatures.

Table 1. Chemical composition of the grey cast iron used in directional solidification experiments $\mathrm{wt} \%$

\begin{tabular}{cccccccc}
\hline $\mathrm{Fe}$ & $\mathrm{C}$ & $\mathrm{Si}$ & $\mathrm{Cr}$ & $\mathrm{Mo}$ & $\mathrm{Mn}$ & $\mathrm{P}$ & $\mathrm{S}$ \\
\hline Bal. & 3.10 & 1.92 & 0.14 & 0.019 & 0.87 & 0.067 & 0.063 \\
\hline
\end{tabular}

\section{Results and discussion}

\subsection{Jmat-Pro calculations on phase transitions}

Fig. 1 shows the Jmat-Pro calculation results for the phase transition in the grey cast iron. With decreasing temperature, the weight fraction of the austenite $(\gamma)$ phase increases gradually during solidification. When it reaches the eutectic temperature, $1155.1^{\circ} \mathrm{C}$, graphite $(\mathrm{G})$ and $\gamma$ are simultaneously precipitated from the liquid (L) until the $\mathrm{L}$ is completely exhausted. After that, the $\mathrm{G}$ begins to precipitate from the oversaturated $\gamma$ phase at temperatures higher than the eutectoid reaction point, $795.4^{\circ} \mathrm{C}$. Lastly, the ferrite $(\alpha)$ and the $\mathrm{G}$ are produced from the $\gamma$ phase via the eutectoid transformation upon further cooling. Therefore, under equilibrium conditions, the phase transition sequence for grey cast iron can be expressed as

$$
\mathrm{L} \rightarrow \mathrm{L}+\gamma \rightarrow \mathrm{L}+\gamma+\mathrm{G} \rightarrow \gamma+\mathrm{G} \rightarrow \gamma+\mathrm{G}+\alpha \rightarrow \alpha+\mathrm{G} .
$$

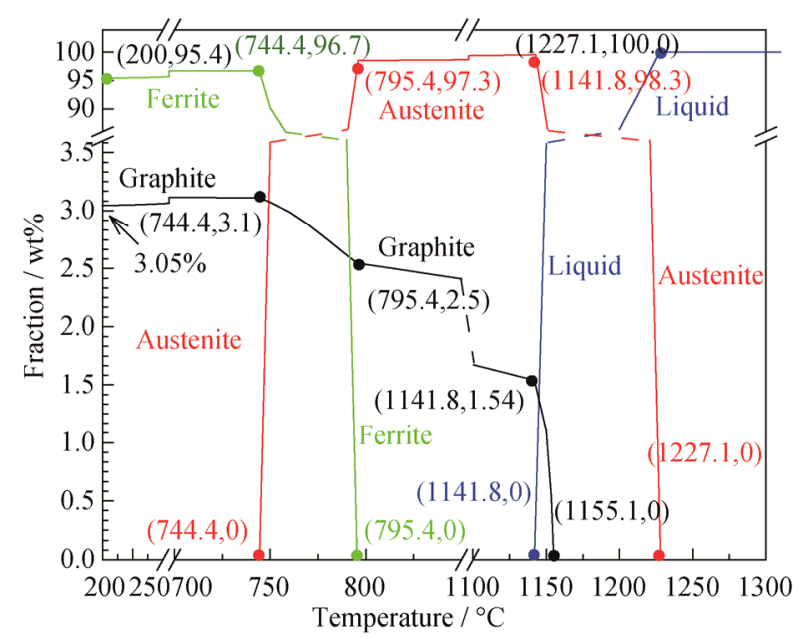

Fig. 1. Jmat-Pro calculation results for the phase transition in grey cast iron.

Notably, the graphite can be formed in three ways: directly nucleated from liquid through the eutectic reaction $(\mathrm{L} \rightarrow \gamma+$ 
G), independently precipitated from the oversaturated $\gamma$ phase $(\gamma \rightarrow \gamma+\mathrm{G})$, and produced by the eutectoid transformation $(\gamma \rightarrow \mathrm{G}+\alpha)$. The graphite is continuously produced before the austenite $\gamma$ phase completely transforms into the ferrite $\alpha$ phase.

\subsection{Microstructure evolution during directional solidi- fication}

Fig. 2 shows the typical longitudinal microstructure of the grey cast iron in the quenched mushy zone during directional solidification. Because the primary austenite $\gamma$ dendrite in the $\mathrm{L}+\gamma$ phase region cannot be observed by $\mathrm{OM}$, the BSE microstructure is inset in the lower-left corner of this figure, even though the contrast between the quenched $\mathrm{L}$ and $\gamma$ phases is not very high because of their similar elemental compositions. As shown in Fig. 2, the face-centered cubic $\gamma$ phase is the primary solidification phase in the grey cast iron, consistent with the observation that the secondary dendritic arms are orthogonal to the primary arms. With decreasing temperature, at least four phase regions, orderly $\mathrm{L}$, $\mathrm{L}+\gamma, \mathrm{L}+\gamma+\mathrm{G}$, and $\gamma+\mathrm{G}$, are observed in the quenched mushy zone. $\mathrm{L}+\gamma+\mathrm{G}$ co-exist in a narrow region behind the $\mathrm{L}+\gamma$ region during solidification, which suggests that the eutectic transformation $\mathrm{L} \rightarrow \gamma+\mathrm{G}$ occurs in this region. All of the phase region distributions and the phase constituents are in good agreement with the calculated results in Fig. 1.

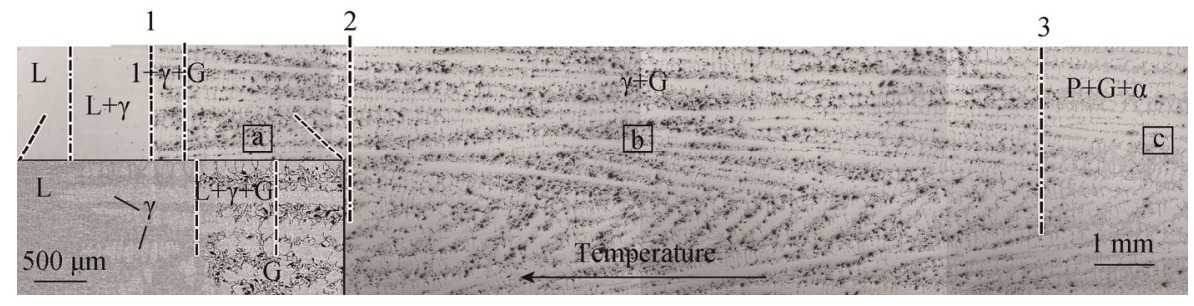

Fig. 2. Longitudinal OM microstructure in the quenched mushy zone of the grey cast iron during directional solidification at a withdrawal rate of $5 \mu \mathrm{m} / \mathrm{s}$; the BSE microstructure in the quenched $(L+\gamma)$-phase region is inset in the lower-left corner of this figure, showing the primary $\gamma$ dendrite morphology and the phase region distribution.

Figs. 3(a), 3(b), and 3(c) show the longitudinal quenched microstructures in the grey cast iron at the lines marked 1,2 , and 3 in Fig. 2, respectively. As shown in Fig. 3(a), the fine graphite initially nucleates at the interface between the interdendritic liquid and the $\gamma$ phase, then grows into the $\gamma$ phase when the liquid is consumed and transformed into the $\gamma$ phase during cooling. Subsequently, the length and the area fraction of the graphite distinctly increase with decreasing temperature, as compared with the graphite microstructures in Figs. 3(a) and 3(b). Some short graphite flakes are precipitated from the $\gamma$-dendrite in the $(\gamma+\mathrm{G})$-phase region, as shown in Fig. 3(b). As presented in Fig. 3(c), a distinct phase interface exists where the $\gamma$ matrix with a light grey contrast is transformed into another matrix structure with a dark grey contrast upon further cooling. The area fraction of graphite in the dark grey region is larger than that in light grey region, suggesting the graphite precipitates when the matrix structure is transformed.

Figs. 4(a), 4(b), and 4(c) show the longitudinal quenched matrix microstructures of the grey cast iron at the locations labeled a, b, and c in Fig. 2, respectively. As shown in Fig. 4(a), in addition to some retained austenite in a light grey contrast, the acicular ferrite and bainite structures combined with the lath-shaped martensite in a dark grey contrast are observed in the $\gamma+\mathrm{G}$ region [8]. We deduced that the high-temperature $\gamma$ phase was transformed into the acicular ferrite and bainite structures combined with lath martensite during quenching at high temperatures, in accordance with others' observations of grey cast iron [9]. As shown in Fig. 4(b), the quenched matrix microstructure is changed from the bainite structure combined with lath-shaped martensite to pearlite $(\mathrm{P})$ structure $\left(\alpha+\mathrm{Fe}_{3} \mathrm{C}\right)$ across the interface [10]. Because the axial temperature gradient during directional solidification, the $\gamma$ matrix was quenched from different temperatures, which leads to different quenched microstructures at different locations in the mushy zone, even within the same phase region of $\gamma+\mathrm{G}$. This effect is likely the reason why the quenched matrix microstructures are different in Figs. 4(a) and 4(b), respectively. As presented in Fig. 4(c), the final matrix microstructure consists of $\mathrm{P}$ with fine laths and a small fraction of granular ferrite $(\alpha)$ formed around the graphite, possibly resulting from the carbon deficit surrounding the graphite precipitates [11-13]. No bainite or austenite structures are observed in the final matrix microstructure. We concluded that the retained $\gamma$ phase, acicular ferrite structure, lath-shaped martensite as well as the bainite were produced by the quenching under fast cooling from the $\gamma$-phase region, whereas pearlite was formed via the directional solidification under a low cooling rate. Thus, the transition of the matrix structure in the grey cast iron can 
be summarized as $\gamma \rightarrow \mathrm{P}\left(\alpha+\mathrm{Fe}_{3} \mathrm{C}\right)+\mathrm{G}+\alpha$, and the last phase region of $\mathrm{P}+\mathrm{G}+\alpha$ should exist at the right side of line 1 in Fig. 2.
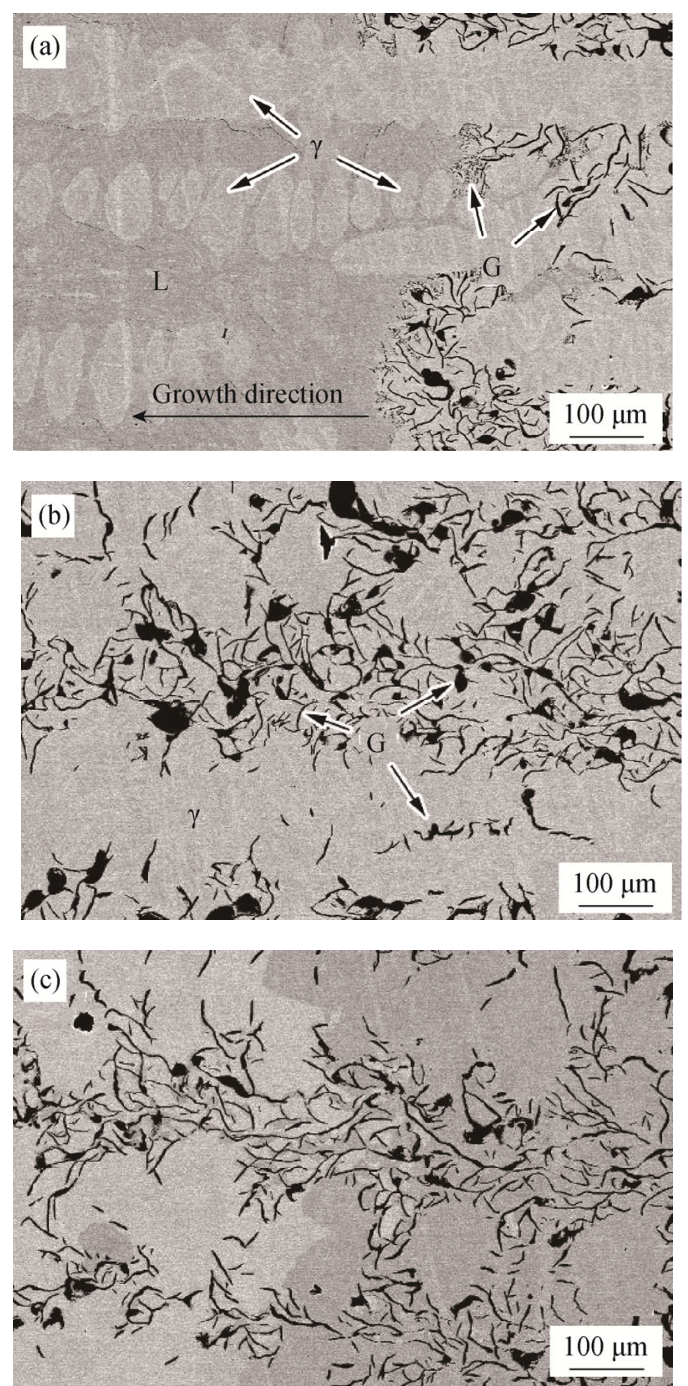

Fig. 3. Longitudinal quenched microstructures in the grey cast iron at line 1 (a), line 2 (b), and line 3 (c) in Fig. 2, respectively.

In summary, at a low cooling rate, the solidification and phase transition sequence for the grey cast iron during cooling should be expressed as $\mathrm{L} \rightarrow \mathrm{L}+\gamma \rightarrow \mathrm{L}+\gamma+\mathrm{G} \rightarrow \gamma+$ $\mathrm{G} \rightarrow \mathrm{P}\left(\alpha+\mathrm{Fe}_{3} \mathrm{C}\right)+\alpha+\mathrm{G}$. For the present grey cast iron with the carbon equivalent (CE) equal to 3.78 , this phase transition sequence is in good agreement with that in the $\mathrm{Fe}-\mathrm{C}$ phase diagram [14-15] and is also in accordance with that calculated using Jmat-Pro, except that the matrix is changed from $\alpha$ to $P$ because of the low cooling rate.

Fig. 5 illustrates the graphite microstructures in the directional solidification region of the grey cast irons at different withdrawal rates. With increasing withdrawal rate, the length of the graphite distinctly decreases. According to EN ISO 945-1 [16], graphite morphologies at the withdrawal rates of 10 and $20 \mu \mathrm{m} / \mathrm{s}$ should be type $\mathrm{D}$, whereas that at $5 \mu \mathrm{m} / \mathrm{s}$ should be type A, as shown in Figs. 5(a), 5(c), and 5(e).
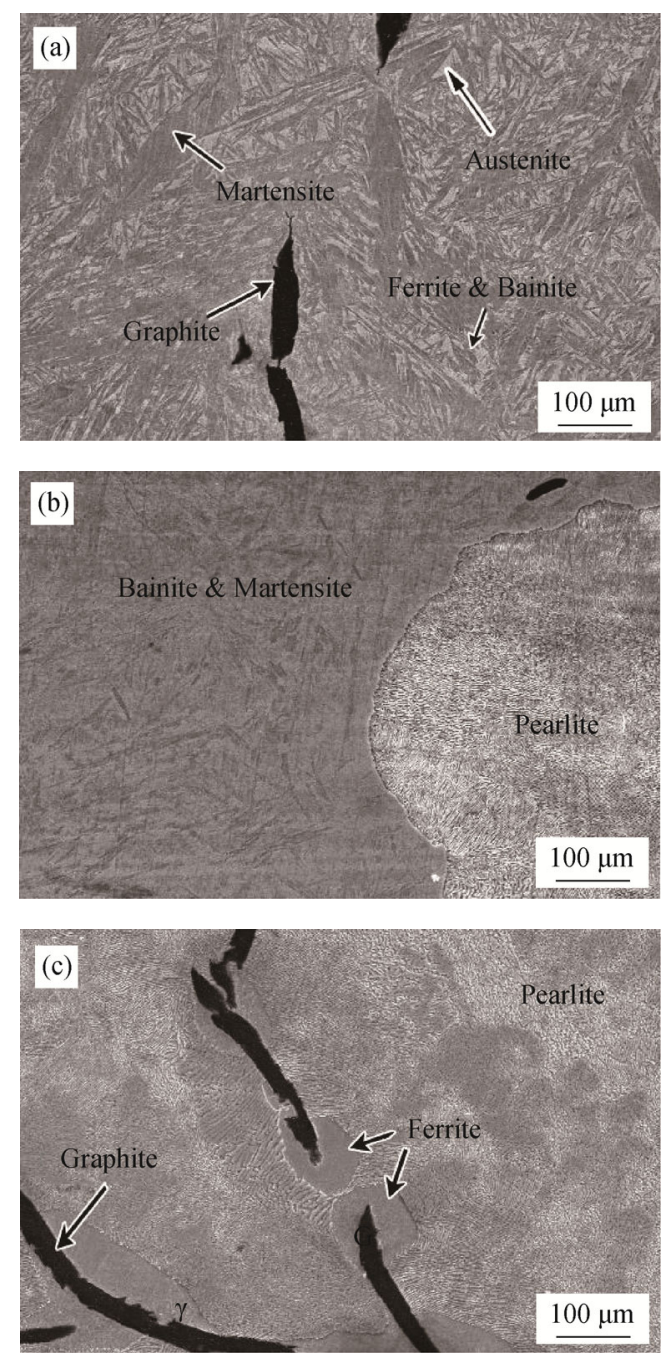

Fig. 4. Longitudinal quenched matrix microstructures of the grey cast iron at locations a (a), b (b), and c (c) labeled in Fig. 2, showing the etched matrix morphologies in the $\gamma+G$ region (a), the neighboring $\gamma \rightarrow P$ transition interface $(b)$, and the $P+\alpha+$ $G$ region (c) after quenching.

The dependences of the area fraction, the length of graphite, and the primary dendrite spacing on the withdrawal rate are presented in Fig. 6. As shown in Figs. 6(a) and 6(b), both the area fraction and the length of the graphite decrease with increasing withdrawal rate. An increase in cooling rate can bring about a departure from equilibrium solidification and increase the undercooling. The carbon in the interdendritic region is easier to reach in a supersaturated solution at high cooling rates. More graphite nucleation sites will be formed, 
and the graphite lacks sufficient time to grow through the diffusion of carbon. Thus, the area fraction and length of graphite particles will decrease with increasing withdrawal rate.
As shown in Fig. 6(c), the primary $\gamma$-dendrite spacing decreases with increasing withdrawal rate, consistent with the relationship in Hunt's primary spacing model [17].
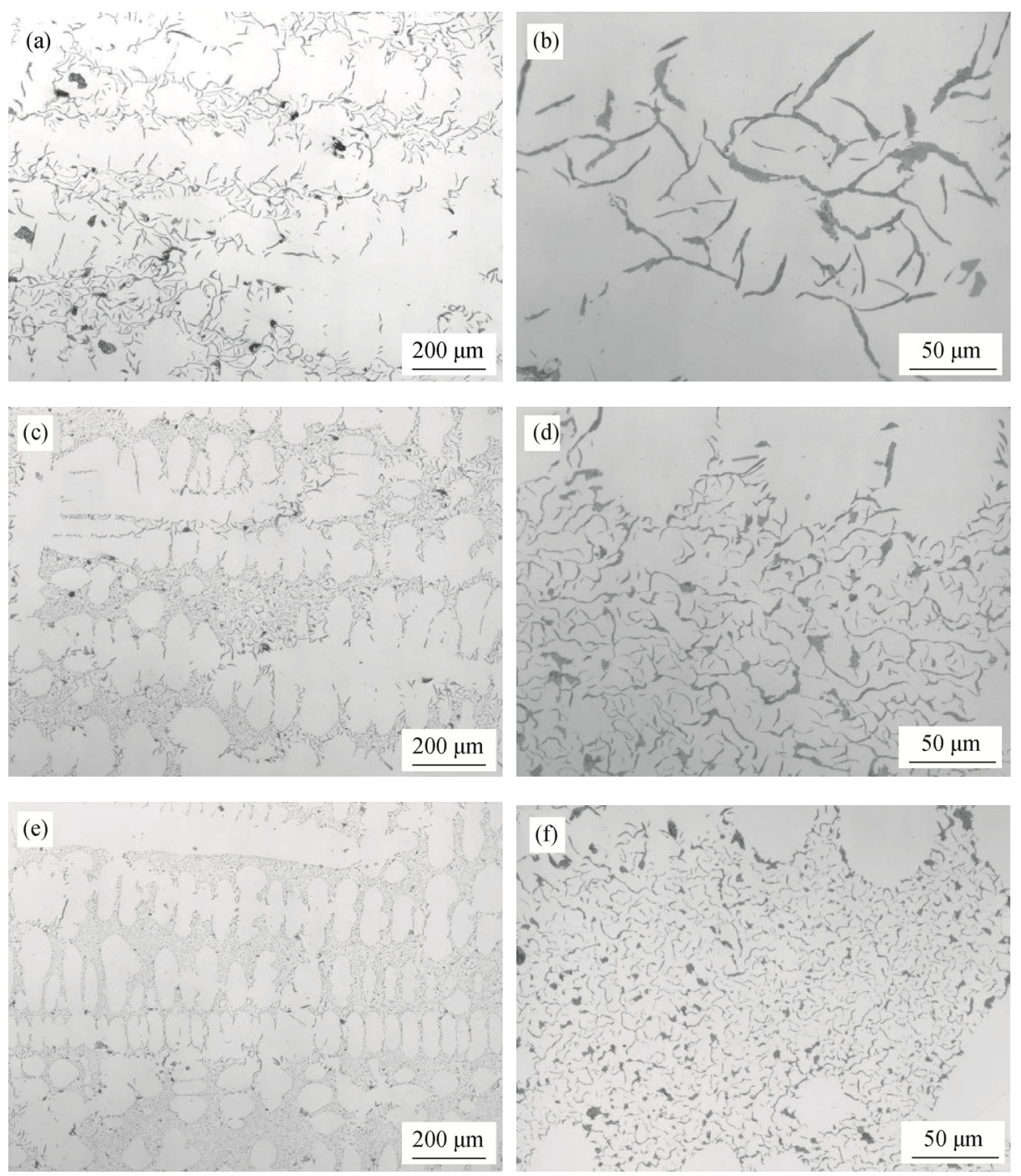

Fig. 5. OM microstructures in the directional solidification region of the grey cast iron, as viewed at low magnification (a, c, and e), and in the interdendritic regions (b, d, and $f$ ) at the withdrawal rates of 5 (a and b), 10 (c and d), and $20 \mu \mathrm{m} / \mathrm{s}$ ( $(\mathrm{e}$ and $\mathrm{f}$ ), respectively.

Fig. 7 shows the typical microstructures neighboring the initial interface of directional solidification at a withdraw rate of $5 \mu \mathrm{m} / \mathrm{s}$. As shown in Fig. 7, a transition region is formed between the as-cast region and the directional solidification region at the start of directional solidification. Induced by the one-dimensional temperature gradient effect, the transition region should be annealed at a graded temperature along the direction of heat flow before the start of directional solidification. The transition region consists of equiaxed $\gamma$ grains but no equiaxed $\gamma$ grain is observed in the as-cast region, which suggests that the transition region should be re-melted or annealed from the $\gamma$-phase region and that these $\gamma$ grains should form by growing or recrystallizing during heating.

Fig. 8 shows the graphite and matrix microstructures in the transition and as-cast regions marked in Fig. 7. The greatest difference in the solidification process between the transition region and the as-cast region is inoculation treatment. As shown in Figs. 8(a) and 8(b), the graphite morphology apparently differs between the transition and as-cast regions. The graphite precipitation was inoculated in the as-cast region during sand casting for the original sample, but not in the transition region when starting directional solidification. In the transition region, the fine and short graphite with a low area fraction is observed mainly in the interdendritic region of the $\gamma$ phase. In the as-cast region, 
however, the coarsened and long graphite particles with a large area fraction are precipitated from the entire $\gamma$ matrix, mainly because the addition of inoculant reduced the supercooling degree of the $\gamma$ matrix and produced the nuclei of the graphite in the $\gamma$ matrix. Induced by the inoculant addition, the location of graphite precipitation is changed from mainly the $\gamma$-interdendritic region to the entire $\gamma$ matrix. This behavior suggests that inoculation can induce heterogeneous nucleation of graphite when the $\gamma$ matrix forms; that is, the inoculation mainly acts on graphite precipitation in the $\gamma$ matrix, not in the liquid or at the solid-liquid front.
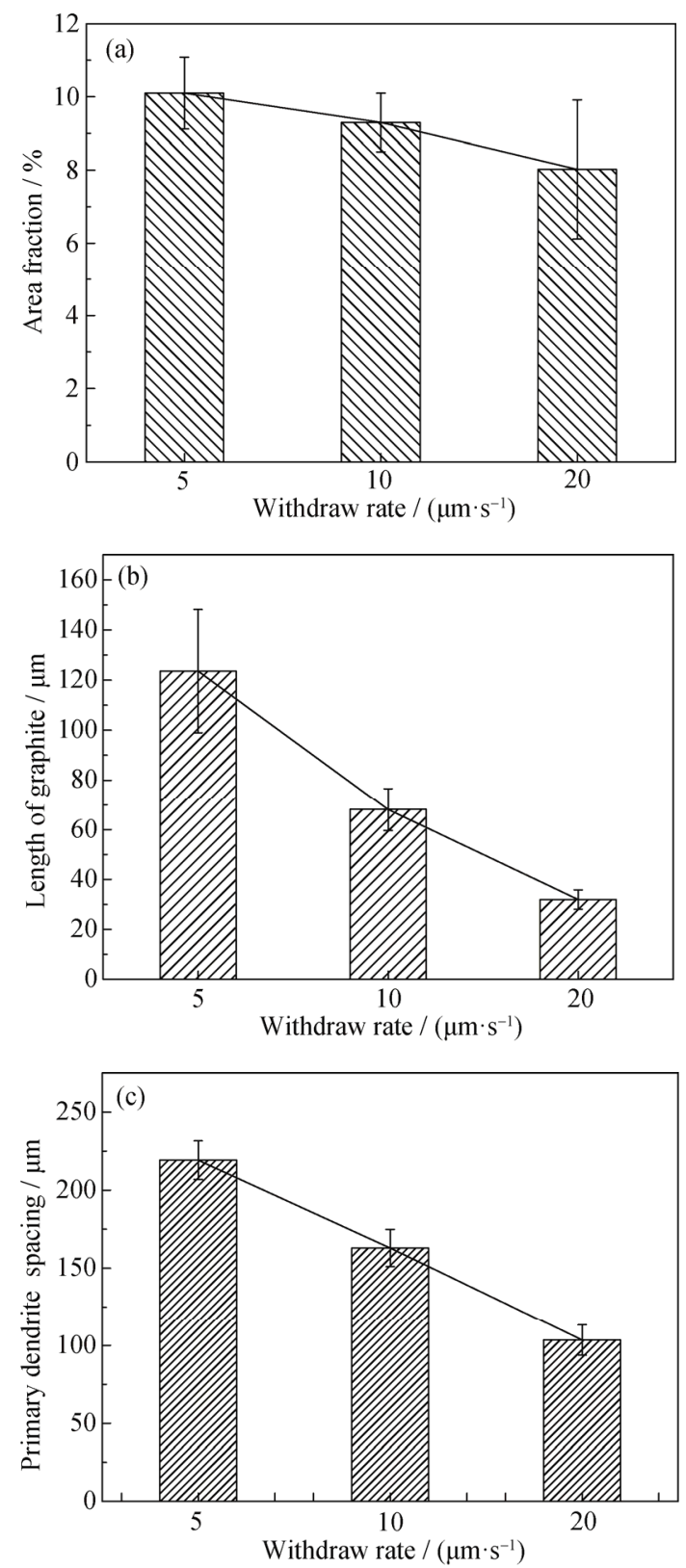

Fig. 6. Dependences of the area fraction of graphite (a), the length of graphite (b), and the primary dendrite spacing (c) on the withdrawal rate.

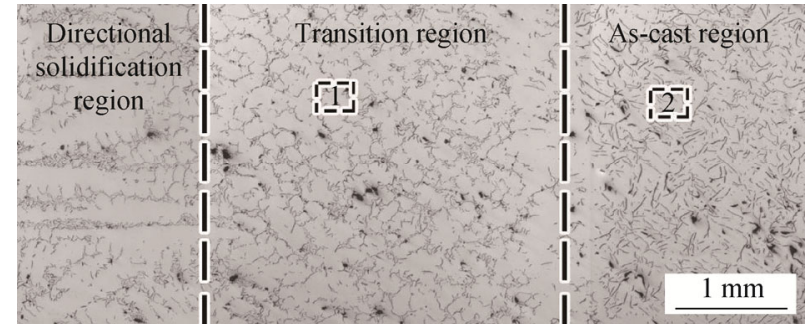

Fig. 7. Typical microstructure neighboring the initial interface of directional solidification at a withdrawal rate of $5 \mu \mathrm{m} / \mathrm{s}$.
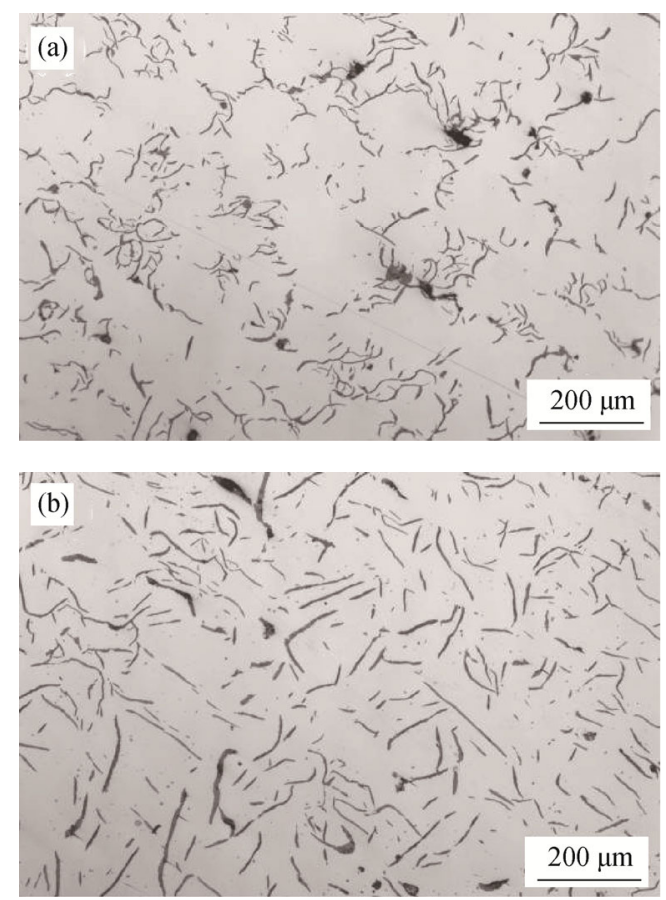

Fig. 8. Graphite and matrix microstructures at different locations marked as 1 and 2 in Fig. 7, showing the graphite morphologies in transition (a) and as-cast (b) regions.

\section{Conclusions}

The solidification characteristics and microstructure evolution in grey iron was investigated through Jmat-Pro simulations and quenching performed during directional solidification. We drew the following conclusions:

(1) The phase transition sequence of grey cast iron is determined as $\mathrm{L} \rightarrow \mathrm{L}+\gamma \rightarrow \mathrm{L}+\gamma+\mathrm{G} \rightarrow \gamma+\mathrm{G} \rightarrow \mathrm{P}(\alpha+$ $\left.\mathrm{Fe}_{3} \mathrm{C}\right)+\alpha+\mathrm{G}$. The graphite can be formed in three ways: directly nucleated from liquid through the eutectic reaction ( $\mathrm{L} \rightarrow \gamma+\mathrm{G})$, independently precipitated from the oversaturated $\gamma$ phase $(\gamma \rightarrow \gamma+\mathrm{G})$, and produced by the eutectoid transformation $(\gamma \rightarrow \mathrm{G}+\alpha)$.

(2) The area fraction and length of graphite as well as the primary dendrite spacing decrease with increase cooling rate. 
Type-A graphite is formed at low cooling rates, whereas a high cooling rate results in type-D graphite.

(3) Induced by the inoculant addition, the location of graphite precipitation is changed from mainly in the $\gamma$ interdendritic region to the entire $\gamma$ matrix.

\section{Acknowledgements}

The authors would like to acknowledge W.Y. Yang and Y.R. Zheng for the assistance of useful discussions. The financial support provided by Ford Motor Company (University Research Program) is also acknowledged.

Open Access This article is distributed under the terms of the Creative Commons Attribution 4.0 International License (http://creativecommons.org/licenses/by/4.0/), which permits unrestricted use, distribution, and reproduction in any medium, provided you give appropriate credit to the original author(s) and the source, provide a link to the Creative Commons license, and indicate if changes were made.

\section{References}

[1] M.M. Hejazi, M. Divandari, and E. Taghaddos, Effect of copper insert on the microstructure of gray iron produced via lost foam casting, Mater. Des., 30(2009), No. 4, p. 1085.

[2] M.M.J. Behnam, P. Davami, and N. Varahram, Effect of cooling rate on microstructure and mechanical properties of gray cast iron, Mater. Sci. Eng. A, 528(2010), No. 2, p. 583.

[3] O. Oloyede, T.D. Bigg, R.F. Cochrane, and A.M. Mullis, Microstructure evolution and mechanical properties of drop-tube processed, rapidly solidified grey cast iron, Mater. Sci. Eng. A, 654(2016), p. 143.

[4] G.L. Rivera, R.E. Boeri, and J.A. Sikora, Solidification of gray cast iron, Scripta Mater., 50(2004), No. 3, p. 331.

[5] M. Hillert, Comments on "Eutectic solidification of gray cast iron”, Scripta Mater., 52(2005), No. 3, p. 249.
[6] X.F. Ding, J.P. Lin, L.Q. Zhang, H.L. Wang, G.J. Hao, and G.L. Chen, Microstructure development during directional solidification of Ti-45Al-8Nb alloy, J. Alloys Compd., 506(2010), No. 1, p. 115.

[7] X.F. Ding, D.F. Liu, P.L. Guo, Y.R. Zheng, and Q. Feng, Solidification microstructure formation in HK40 and HH40 alloys, Int. J. Miner. Metall. Mater., 23(2016), No. 4, p. 442.

[8] A. Vadiraj, G. Balachandran, and M. Kamaraj, Structure and property studies on austempered and As-cast ausferritic gray cast irons, J. Mater. Eng. Perform., 19(2010), No. 7, p. 976.

[9] W. Xu, M. Ferry, and Y. Wang, Influence of alloying elements on as-cast microstructure and strength of gray iron, Mater. Sci. Eng. A, 390(2005), No. 1-2, p. 326.

[10] L. Collini, G. Nicoletto, and R. Konečná, Microstructure and mechanical properties of pearlitic gray cast iron, Mater. Sci. Eng. A, 488(2008), No. 1-2, p. 529.

[11] M.H. Cho, S.J. Kim, R.H. Basch, J.W. Fash, and H. Jang, Tribological study of gray cast iron with automotive brake linings: The effect of rotor microstructure, Tribol. Int., 36(2003), No. 7, p. 537.

[12] M. Ramadan, M. Takita, and H. Nomura, Effect of semi-solid processing on solidification microstructure and mechanical properties of gray cast iron, Mater. Sci. Eng. A, 417(2006), No. 1-2, p. 166.

[13] M.L. Wu, F.W. Guo, M. Li, and Y.F. Han, Effect of trace strontium addition on microstructure and room temperature fracture toughness of $\mathrm{Nb}-12 \mathrm{Si}-22 \mathrm{Ti}$ alloys, Mater. Sci. Forum, 849(2016), p. 603.

[14] T.B. Massalski, Binary Alloy Phase Diagrams, Edited by H. Okamoto, P.R. Subramanian, and L. Kacprzak, American Society for Metals International, Metals Park, Ohio, USA, 1990, p. 3589.

[15] D. Holmgren, A. Diószegi, and I.L. Svensson, Effects of carbon content and solidification rate on the thermal conductivity of grey cast iron, Tsinghua Sci. Technol., 13(2008), No. 2, p. 170.

[16] EN ISO 945-1: 2010, Microstructure of Cast Irons-Part 1: Graphite Classification by Visual Analysis, EU, 2010.

[17] J.D. Hunt and S. Lu, Numerical modeling of cellular/dendritic array growth: spacing and structure predictions, Metall. Mater. Trans. A, 27(1996), No. 3, p. 611. 